\title{
Analyzing Track Responses to Train Braking
}

\author{
Bose, Tulika; Levenberg, Eyal; Zania, Varvara
}

Published in:

Proceedings of the Institution of Mechanical Engineers, Part F: Journal of Rail and Rapid Transit

Link to article, DOI:

10.1177/0954409718761242

Publication date:

2018

Document Version

Peer reviewed version

Link back to DTU Orbit

Citation (APA):

Bose, T., Levenberg, E., \& Zania, V. (2018). Analyzing Track Responses to Train Braking. Proceedings of the Institution of Mechanical Engineers, Part F: Journal of Rail and Rapid Transit, 232(7), 1984-1993. https://doi.org/10.1177/0954409718761242

\section{General rights}

Copyright and moral rights for the publications made accessible in the public portal are retained by the authors and/or other copyright owners and it is a condition of accessing publications that users recognise and abide by the legal requirements associated with these rights.

- Users may download and print one copy of any publication from the public portal for the purpose of private study or research.

- You may not further distribute the material or use it for any profit-making activity or commercial gain

- You may freely distribute the URL identifying the publication in the public portal 


\section{Analyzing Track Responses to Train Braking}

\section{Tulika Bose}

Department of Civil Engineering, Technical University of Denmark

Nordvej, Building 119

Kgs. Lyngby 2800, Denmark

Email: tulbo@byg.dtu.dk

Eyal Levenberg (corresponding author)

Department of Civil Engineering, Technical University of Denmark Nordvej, Building 119

Kgs. Lyngby 2800, Denmark

Tel: +45 4525 1907; Email: eylev@byg.dtu.dk

\section{Varvara Zania}

Department of Civil Engineering, Technical University of Denmark Nordvej, Building 119

Kgs. Lyngby 2800, Denmark

Email: vaza@byg.dtu.dk 


\section{ABSTRACT}

The objective of this study was to suggest a response-analysis framework for railway tracks subjected to braking. An analytical formulation was developed, in which the rail-track system was modeled as an infinite beam supported by an orthogonal Winkler foundation consisting of linear springs in perpendicular directions. The spring constants were varied over a wide range in order to represent different track types. Braking loads were simulated as representative sets of vertical and longitudinal forces, either concentrated or distributed. Considering a realistic set of model parameters, the approach was demonstrated by evaluating track responses for a single axle and for a full train. The computations included determination of axial rail stresses, forces at the base of a sleeper, and the associated friction demand required to resist longitudinal slippage. Based on these analyses, it is concluded that longitudinal track responses have a much longer influence zone compared to vertical track responses. This implies that calculations involving a full train must be done on a case-by-case basis, i.e., they cannot be deduced from a single axle analysis. It is also found that high values of friction demand may develop at the sleeper bases - indicating possible slippage. Overall, the proposed formulation provides a highly adaptable and easily implementable first-order mechanistic tool for analysis of track responses to decelerating vehicular loads.

Keywords: Railway, Train Braking, Longitudinal loads, Winkler foundation, Friction demand.

\section{Introduction}

Over the years, the rail industry has been facing constant public and economic demands to expand service, especially in accommodating faster and heavier trains, and increased line capacities. ${ }^{1}$ This motivated improvements in vehicles, e.g., powering individual railcars for increased acceleration and top speed, ${ }^{2}$ and developing newer/stronger braking systems. ${ }^{3,4}$ The demands also triggered advancements to the physical infrastructure, both within the realm of traditional ballasted tracks, e.g., incorporating new sleeper designs ${ }^{5,6}$ and upgrading vibration absorbing components, ${ }^{7-9}$ as well as by exploring non-traditional track types, e.g., reinforcing ballast layers, ${ }^{10}$ replacing subballast with asphalt concrete, i.e., asphalt underlayment solution, ${ }^{11-13}$ 
and switching to ballastless track based on Portland cement concrete, i.e., slab track solution. ${ }^{14}$ Another ballastless track-type that has been gaining recent attention is asphalt overlayment. ${ }^{15-17}$

An essential step in accepting new track concepts or new vehicles (or both), before fullscale adaptation within the live network, is evaluating mechanical infrastructure responses (i.e., forces, stresses, displacements, strains) to anticipated train loads. As an integral part of such evaluation, the current work focuses on analyzing responses to loads exerted during a braking event. Doing so is deemed a priority especially for asphalt overlayment solutions wherein precast sleepers are placed on an asphalt pavement surface - possibly without crib or shoulder ballast. Therefore, at the superstructure-substructure interface, longitudinal loads are solely counteracted by the sliding resistance available at the sleeper bases. This resistance may be inferior compared to a ballasted track where crib and shoulder ballast also contribute to the sliding resistance. ${ }^{18,19}$ Analyzing track responses due to braking is also warranted in view of advances in braking systems. The traditional method of slowing or completely stopping trains involves clamping of the wheels. In this case, the braking effort is limited by the wheel-rail friction conditions, which depend on factors such as cleanliness level, and moisture conditions; ${ }^{20}$ it is also influenced by railhead roughness. ${ }^{21}$ For this reason, so-called track-brakes were devised, in which the braking force is applied to the top surface of the rails between the axles over a certain length. These operate either through friction with the rail or by generating eddy currents in the rails, creating forces acting in the direction opposite to train movement. ${ }^{3}$

Analysis of track responses to train braking requires modeling the effects of both vertical and longitudinal vehicular loads. While modeling the former category is fairly established in railway design, only a handful of studies were identified to address the latter. $\operatorname{Van}^{22}$ set forth to numerically study thermal loads on rails and bridges as well as mechanical loads due to braking or accelerating trains. In the proposed model, the two rails were jointly treated as a single beam connected to longitudinal elasto-plastic springs with an assumed (fixed) yield limit. The beam's axial stresses and displacements were then evaluated under varying temperature conditions and also under the influence of an applied longitudinal load. The latter was uniformly distributed, representing the effect of a braking (or accelerating) train, averaged over its entire length. Some shortcomings of the study are that the effects at individual sleepers or due to distinct axles were not included; also, interaction between longitudinal and vertical loading directions was not accounted for. 
The work of Rhodes et al. ${ }^{23}$ was motivated by a number of cases where track structures experienced sleeper movements relative to rails and ballast. These resulted from high traction forces induced by heavy freight trains. The study included field measurements of axial rail stresses and offered some design guidelines. However, no mechanistic model was offered, capable of providing quantitative explanation to the observed field responses. Zhang et al. ${ }^{24}$ carried out finite element analysis to investigate the longitudinal forces within the rail fastening system and the friction demand at the rail rail-pad interfaces during train acceleration. All frictional contact interfaces within the track components were modeled as Coulomb-type; in particular, a value of 0.70 was taken for the sleeper-substructure interface. A wheel was accelerated in the model with an assumed wheel-rail friction coefficient of 0.5 . Their analysis found that longitudinal forces at the rail rail-pad interfaces were almost zero in front of the wheel. It was also reported that only about five sleepers behind the accelerating wheel participated in carrying the longitudinal load. The paper did not provide a physical explanation of this non-symmetric distribution of responses. Additionally, relative slippage between components and development of residual stresses in the system were not considered.

Subsequently, the objective of this study is to develop a model suited for analyzing the response of railway tracks to braking events, accommodating different braking systems. The purpose is to provide a mechanistic portrayal of how decelerating vehicular loads are handled within a track superstructure. Specifically, the model should allow: (i) resolving the axial stresses along the rail; (ii) identifying the number of participating sleepers and their relative contribution in transferring the braking-related loads to the substructure; and (iii) describing the friction demand at the sleeper-substructure level (or equivalently at the rail-railpad interfaces). An analytic framework is sought for the development in order to provide closed-form solutions that are easily implementable, fast to compute, and therefore highly adaptable as a first-order engineering tool.

The paper commences by introducing the basic model equations and assumptions; this is followed by demonstrating calculations and interpreting results for the case of a single axle and for a full-length train. This is done assuming a traditional braking system while employing a range of model parameters representing different track-types.

\section{Response modeling}

In this section, a new two-dimensional response-model for railway tracks is proposed, with the focus on vehicular loads generated during braking. Consider, as shown in Figure 1, a single 
train axle decelerating over a rail, supported on sleepers that are resting on a substructure. The sleepers are all identical, sequentially numbered and spaced at an interval of $s$. A Cartesian coordinate system is shown with its origin placed at the rail surface directly above the $i^{\text {th }}$ sleeper (i.e., a random sleeper being evaluated within the braking zone). The $Z$ axis points downward and the $X$ axis points along the travel direction. The axle exerts a vertical load with magnitude $2 P_{Z}$ representing its total weight and concurrently a longitudinal load of magnitude $2 P_{X}$ signifying its braking effort. The resulting vertical and longitudinal forces at the base of the $i^{\text {th }}$ sleeper are denoted as $S_{Z}$ and $S_{X}$ respectively. Equilibrium considerations dictate that the same forces must also operate at the rail-railpad interface (i.e., fastening assembly).

\section{“[insert Figure 1.]"}

The model of the physical problem described in Figure 1 is presented in Figure 2 by referring to a single axle side, i.e., a decelerating single wheel. The rail is considered as an infinite homogenous Euler-Bernoulli beam with cross-sectional area $A$, Young's modulus $E$, and moment of inertia $I$. The weight per unit length of the beam is taken as: $\gamma=\gamma_{R}+W / s$ wherein $\gamma_{R}$ is the rail weight per unit length, and $W / s$ is the distributed weight considering one-half of a sleeper implying $2 W$ denotes a full sleeper weight. The neutral axis of the beam, passing through the geometric centroid of the cross section, is denoted by the dashed horizontal line.

\section{“[insert Figure 2.]"}

A Cartesian coordinate system is positioned at the point of evaluation corresponding to the location of the $i^{\text {th }}$ sleeper, in analogy to Figure 1 ; the origin coincides with the neutral axis. Loads exerted by the wheel, $P_{Z}$ and $P_{X}$, are applied to the neutral axis at a distance $x$ from the coordinate origin. The beam is supported by an orthogonal Winkler type foundation, which includes both vertical and longitudinal linear springs at right angles, connected to the beam along the neutral axis. The springs are shown at discrete locations, only for illustration purposes, in effect they are continuous. The corresponding spring constants are $k_{Z}$ and $k_{X}$, both with units of force per length squared; they represent the cumulative support offered to the rail by all underlying track components, with magnitudes depending on the track-type. 
The vertical beam displacement at the coordinate origin $\left(u_{z}\right)$ is expressed following Hetenyi $^{25}$ as:

$$
u_{Z}=\frac{P_{Z} \beta_{Z} e^{-\beta_{Z}|x|}}{2 k_{Z}}\left(\cos \beta_{Z} x+\sin \beta_{Z}|x|\right)
$$

wherein:

$$
\beta_{Z}=\sqrt[4]{\frac{k_{Z}}{4 E I}}
$$

Accordingly, the vertical force at the base of the $i^{\text {th }}$ sleeper including self-weight $\left(S_{Z}\right)$ can be approximated as:

$$
S_{Z}=s\left(u_{Z} k_{Z}+\gamma\right)=\frac{s P_{Z} \beta_{Z} e^{-\beta_{Z}|x|}}{2}\left(\cos \beta_{Z} x+\sin \beta_{Z}|x|\right)+s \gamma
$$

In this expression, the continuous support model is employed for evaluating the response at a discrete location, i.e., a sleeper. The error involved in such approximation has been verified to be negligibly small by comparison against an exact analytic solution for a periodically supported beam. $^{26}$

For determining responses in the longitudinal direction, Figure 3 presents a magnified view of an infinitesimal beam element of length $d x$ located near, and to the left of, the coordinate origin (refer to Figure 2). This element experiences axial stress along its neutral axis, having magnitude $\sigma_{N}$ at the origin and $\sigma_{N}+d \sigma_{N}$ at a distance $x+d x$ from $P_{X}$. The chosen sign convention is such that positive stress or strain indicates compression. Also, the element is displaced by $u_{X}$ in the direction of $P_{X}$; this activates the longitudinal springs and induces an opposing axial stress along the neutral axis with magnitude $k_{X} u_{X}$.

\section{“[insert Figure 3.]"}

Under the abovementioned stress conditions, longitudinal force-equilibrium dictates:

$$
u_{X}=\frac{A}{k_{X}} \frac{d \sigma_{N}}{d x}
$$


This expression provides a link between the displacement $u_{X}$ and the change in $\sigma_{N}$ as $x$ changes. Alternatively, the displacement $u_{X}$ can be viewed as the summation of all deformation increments (at the origin) as the force $P_{X}$ 'moves' from infinity towards $x$. This situation is expressed by the integral:

$$
u_{X}=\int_{\infty}^{x} \frac{\sigma_{N}}{E} d x
$$

Combining equations (4) and (5) gives the governing integro-differential equation:

$$
\frac{A E}{k_{X}} \frac{d \sigma_{N}}{d x}=\int_{\infty}^{x} \sigma_{N} d x
$$

This equation is solved with a boundary condition stating that for $x=0^{+}$the axial stress at the coordinate origin is $\sigma_{N}=-0.5 P_{X} / A$ (tensile) and for $x=0^{-}$the axial stress at the coordinate origin is $\sigma_{N}=0.5 P_{X} / A$ (compressive). The solution is therefore:

$$
\sigma_{N}=-\frac{P_{X} \operatorname{sgn}(x)}{2 A} e^{-\beta_{X}|x|}
$$

wherein $\operatorname{sgn}(\cdot)$ is the signum function, and:

$$
\beta_{X}=\sqrt{\frac{k_{X}}{E A}}
$$

Utilizing either equation (4) or equation (5), an expression representing the longitudinal beam displacement at the coordinate origin is:

$$
u_{X}=\frac{\beta_{X} P_{X}}{2 k_{X}} e^{-\beta_{X}|x|}
$$

from which the longitudinal force at the base of the $i^{\text {th }}$ sleeper $\left(S_{X}\right)$ can be approximated as:

$$
S_{X}=s u_{X} k_{X}=\frac{s P_{X} \beta_{X}}{2} e^{-\beta_{X}|x|}
$$

Similar to the vertical case, the suitability of employing a continuous horizontal support model for analysis at a discrete location, was verified by means of a separate finite element analysis. 
This longitudinal force is transferred from the rail via the fastener-assembly to the sleepers, with the analysis focusing on the interface with the substructure. Overall, the spring forces were derived by neglecting any second order effects arising from coupling of responses in vertical and longitudinal directions.

Based on equations (3) and (10), an expression describing the friction demand $\mu_{D}$ at the base of the $i^{\text {th }}$ sleeper is:

$$
\mu_{D}=\frac{S_{X}}{S_{Z}}=\frac{P_{X} \beta_{X} e^{-\beta_{X}|x|}}{P_{Z} \beta_{Z} e^{-\beta_{Z}|x|}\left(\cos \beta_{Z} X+\sin \beta_{Z}|x|\right)+2 \gamma}
$$

At its core, $\mu_{D}$ represents the required resistance at the sleeper base to ensure no-slip conditions during a braking event. As can be seen $\mu_{D}$ is a function of both the position and intensity of loading; it also depends on all model parameters including sleeper spacing (via the expression for $\gamma$ ). A closed form solution for the peak value of the friction demand $\mu_{D}^{\max }$ or the corresponding load position cannot be presented; however, for a restricted domain of model parameters $\mu_{D}^{\max }$ may be approximated by assuming it develops where the vertical force $S_{Z}$ is minimal, i.e., for $x=\pi / \beta_{Z}$

If the friction demand cannot be realistically matched, then sliding takes place at the sleeper bases. This sliding gives rise to redistribution of longitudinal forces and development of residual stresses within the superstructure. Such inelastic behavior violates the basic assumptions of the proposed framework, rendering it inapplicable.

As means of addressing modern braking technologies, there is a need to provide a solution for a distributed loading both in the vertical and longitudinal directions. This is because the braking effort may not rely solely on a wheel clamping mechanism but apply over a certain rail length. Therefore, the model is hereafter extended to consider the case of vertical and longitudinal loads with magnitudes $P_{Z}^{\mathrm{mb}}$ and $P_{X}^{\mathrm{mb}}$ respectively, each acting over a length $x_{0}$ (superscript $\mathrm{mb}$ is introduced to indicate 'modern braking'). The responses at the origin become: 


$$
\begin{aligned}
& u_{Z}^{\mathrm{mb}}=\frac{P_{Z}^{\mathrm{mb}}}{2 k_{Z} x_{0}}\left(\begin{array}{l}
e^{\beta_{Z}\left(x+0.5 x_{0}\right)} \cos \left(\beta_{Z}\left(x+0.5 x_{0}\right)\right)-e^{\beta_{Z}\left(x-0.5 x_{0}\right)} \cos \left(\beta_{Z}\left(x-0.5 x_{0}\right)\right) \\
+H\left(x+0.5 x_{0}\right)\left(2-\cos \left(\beta_{Z}\left(x+0.5 x_{0}\right)\right)\left(e^{-\beta_{Z}\left(x+0.5 x_{0}\right)}+e^{\beta_{Z}\left(x+0.5 x_{0}\right)}\right)\right) \\
-H\left(x-0.5 x_{0}\right)\left(2-\cos \left(\beta_{Z}\left(x-0.5 x_{0}\right)\right)\left(e^{-\beta_{Z}\left(x-0.5 x_{0}\right)}+e^{\beta_{Z}\left(x-0.5 x_{0}\right)}\right)\right)
\end{array}\right) \\
& \sigma_{N}^{\mathrm{mb}}=-\frac{P_{X}^{\mathrm{mb}} \operatorname{sgn}(x)}{2 A \beta_{X} x_{0}}\left(\begin{array}{l}
e^{\beta_{X}\left(x+0.5 x_{0}\right)}-e^{\beta_{X}\left(x-0.5 x_{0}\right)}-H\left(x-0.5 x_{0}\right)\left(2-e^{-\beta_{X}\left(x-0.5 x_{0}\right)}-e^{\beta_{X}\left(x-0.5 x_{0}\right)}\right) \\
+H\left(x+0.5 x_{0}\right)\left(2-e^{-\beta_{X}\left(x+0.5 x_{0}\right)}-e^{\beta_{X}\left(x+0.5 x_{0}\right)}\right)
\end{array}\right) \\
& u_{X}^{\mathrm{mb}}=\frac{P_{X}^{\mathrm{mb}}}{2 k_{X} x_{0}}\left(\begin{array}{l}
e^{\beta_{X}\left(x+0.5 x_{0}\right)}-e^{\beta_{X}\left(x-0.5 x_{0}\right)}-H\left(x-0.5 x_{0}\right)\left(2-e^{-\beta_{X}\left(x-0.5 x_{0}\right)}-e^{\beta_{X}\left(x-0.5 x_{0}\right)}\right) \\
+H\left(x+0.5 x_{0}\right)\left(2-e^{-\beta_{X}\left(x+0.5 x_{0}\right)}-e^{\beta_{X}\left(x+0.5 x_{0}\right)}\right)
\end{array}\right)
\end{aligned}
$$

wherein the coordinate $x$ refers to the (lateral) distance between the evaluation point and the center of the load distribution, $H(\bullet)$ is the Heaviside step function, and all other symbols as previously defined. It is noted that at the limit, as $x_{0} \rightarrow 0$, the above expressions reduce to the point load expressions.

From the displacement expressions, equations (12) and (14), the friction demand in the case of a modern braking system can be evaluated (at the base of a sleeper) following a similar approach as described above, i.e., calculating the forces $S_{X}$ and $S_{Z}$, and then their ratio (see equation (11)). The basic analysis in this case deals with a single wagon, given that there is a contribution to $S_{X}$ and $S_{Z}$ from the axles as well as from the distributed braking unit. Thus, all the above expressions, jointly, can be instrumental in quantifying superstructure responses under braking events, for both traditional and modern braking systems.

\section{Analysis of track response}

The purpose of this section is to demonstrate model capabilities for evaluating the braking event of a single axle and then of a full train. Only the point force solutions were considered representing a traditional braking system. A loading ratio of $P_{X} / P_{Z}=0.25$ was chosen given that it represents an accepted and realistic wheel-rail friction level for dry conditions. ${ }^{27-29}$ The properties of the beam were chosen as per UIC 60 rail section. A standard concrete sleeper was selected with 
weight $2 W=2845 \mathrm{~N}$ (i.e., mass of $290 \mathrm{~kg}$ ); and spacing as $s=600 \mathrm{~mm}$ (therefore, $\gamma=2.96 \mathrm{~N} / \mathrm{mm}$ ) .

To represent different support conditions, a range of spring constants were chosen in vertical and longitudinal directions. The former were considered, from a relatively low value of 20 MPa to a high value of $100 \mathrm{MPa} .{ }^{30,31}$ Similarly, the longitudinal spring constants were selected over a wide range of $5 \mathrm{MPa}$ to $30 \mathrm{MPa}$. This choice was deemed reasonable based on measured force-displacement behavior during sleeper pullout tests ${ }^{18}$ from which longitudinal spring constants in the range of $6 \mathrm{MPa}$ to $13 \mathrm{MPa}$ were extracted. Additionally, in connection with track resistance to longitudinal displacement, similar values are mentioned in the UIC Code dealing with track-bridge interactions. ${ }^{28}$

\section{Braking of a single axle}

Figure 4 presents model responses for a single axle with $2 P_{Z}=170 \mathrm{kN}$ and $2 P_{X}=42.5 \mathrm{kN}$ . Four charts are shown, each depicting a different response parameter as a function of the load position $(x)$. For graphical presentation purposes, the abscissas in the charts span different ranges of $x$. Figures 4(a) and 4(b) illustrate forces in vertical $\left(S_{Z}\right)$ and longitudinal $\left(S_{X}\right)$ directions, induced at the base of the $i^{\text {th }}$ sleeper, as per equations (3) and (10) respectively. It can be seen that the average peak vertical and longitudinal forces differ by almost two orders of magnitude: $32 \mathrm{kN}$ vs. $0.6 \mathrm{kN}$. The displacements corresponding to these average peak force levels are $1.0 \mathrm{~mm}$ and $0.07 \mathrm{~mm}$ respectively.

In addition, forces at the base of a sleeper depend on the spring constant values; smaller spring constants correspond to lower peak forces and a wider region of load influence. Conversely, larger spring constants imply higher peak forces and narrower zone of load influence. Vertical forces are observed to decay much faster (from their peak level) with respect to $x$ as compared to

longitudinal forces. The impact of vertical load becomes almost negligible as $|x|>2 \mathrm{~m}$, i.e., about three sleeper spacings, while the longitudinal load influences a range of $|x|>18 \mathrm{~m}$, i.e., almost 30 sleeper spacings.

Figure 4(c) presents the axial stresses along the beam's neutral axis $\left(\sigma_{N}\right)$ at the coordinate origin as per equation (7). It can be observed that the stress reverses sign with respect to the load 
position; $\sigma_{N}$ is compressive for negative values of $x$ and is tensile for positive $x$ 's. The reversal occurs at $x=0$ and the peak longitudinal stresses are attained when the load is closest to the origin (just before and just after). The peak stress is independent of the support condition, but the rate of decay with $x$ is influenced by $k_{X}$. It is noted that the peak longitudinal stress magnitude is relatively small and constitutes less than $5 \%$ of the maximal bending stress caused by $P_{z}$.

\section{"[insert Figure 4.]"}

Finally, Figure 4(d) presents the friction demand at the base of a sleeper $\left(\mu_{D}\right)$, as per equation (11). It can be seen, that $\mu_{D}$ is symmetric about the loading location, consistent with the responses of both vertical and longitudinal forces. As the loading approaches a sleeper from a negative $x$, the friction demand increases until $\mu_{D}^{\max }$ is attained. Then after $\mu_{D}$ begins to decrease in value until the load is directly above the sleeper. With further increase in $x$, the above described behavior is mirrored, i.e., the friction demand rises to $\mu_{D}^{\max }$ and then decreases towards zero as $x \rightarrow \infty$. With reference to Figure 4(a) it can be seen that the $x$ corresponding to $\mu_{D}^{\max }$ is nearly the $x$ corresponding to minimal vertical force (i.e., locations where upward beam bending counteracts self-weight). The results presented in Figure 4 indicate a general trend of increasing $\mu_{D}^{\max }$ with increasing spring constants. It is noted that $\mu_{D}^{\max }$ values larger than unity may be too high to be provided by a purely frictional mechanism at the sleeper-substructure interface. ${ }^{32,33}$

Figure 5 further investigates the dependence of $\mu_{D}^{\max }$ on the spring constants for a single axle. The considered loads for this case remained: $2 P_{Z}=170 \mathrm{kN}$ and $2 P_{X}=42.5 \mathrm{kN}$. Two charts are presented, depicting the variation of $\mu_{D}^{\max }$ as a function of the ratio $k_{Z} / k_{X}$ within the range of 1.5 to 4.5; this was done for $k_{X}$ in the range of $5 \mathrm{MPa}$ to $30 \mathrm{MPa}$. In Figure 5(a) this information is shown for a standard sleeper with mass of $290 \mathrm{~kg}$ while in Figure 5(b) a heavier sleeper was considered with a mass of $590 \mathrm{~kg}$. Within this analysis domain, $\mu_{D}^{\max }$ may be approximated to a relative error of $0.5 \%$ by inserting $x=\pi / \beta_{Z}$ in equation (11). 
Referring to Figure 5(a), it can be seen that $\mu_{D}^{\max }$ exhibits a positive relationship with both $k_{X}$ and $k_{Z}$, and is governed by the ratio of $k_{Z} / k_{X}$. The sensitivity of $\mu_{D}^{\max }$ to increase in $k_{Z} / k_{X}$ is dependent on the value of $k_{X}$. At low $k_{X}$ values $\mu_{D}^{\max }$ is mildly sensitive to increase in $k_{Z}$; conversely, at higher values of $k_{X}$ the influence of increase in $k_{Z} / k_{X}$ becomes more pronounced. Low values of $k_{X}$ imply small horizontal forces at the base of a sleeper. For the chosen range of $k_{Z} / k_{X}$, these small forces mostly govern the maximal friction demand. High values of $k_{X}$ are associated with larger longitudinal forces at the base of a sleeper. At the same time, within the considered $k_{z} / k_{X}$ range, larger $k_{Z}$ values imply lower vertical force (due to upward beam bending counteracting self-weight). Hence, for these positions, it is the adverse combination of high longitudinal force and low vertical force, which governs the peak friction demand.

Referring to Figure 5(b), it can be seen that $\mu_{D}^{\max }$ also exhibits a positive relationship with both $k_{X}$ and $k_{Z}$, and is governed by the ratio of $k_{Z} / k_{X}$. However, with respect to Figure 5(a), the behavior appears different; $\mu_{D}^{\max }$ is mildly sensitive to $k_{Z} / k_{X}$ across the entire range of considered $k_{X}$ values. It is important to note that the $\mu_{D}^{\max }$ levels in Figure 5(b) are much smaller than those in Figure 5(a), and are well within acceptable levels of interface friction. The above features are a direct result of employing a heavier sleeper.

\section{Braking of a full train}

The track response model is hereafter demonstrated by analyzing a braking event involving a full train. Thalys HST train was selected for this purpose; ${ }^{34}$ it includes two locomotives and eight carriages, having total of 26 axles spanning a length of approximately $200 \mathrm{~m}$. For the different train units, Table 1 denotes each axle by an index $j(j=1 \ldots 26)$ such that $j=1$ and $j=26$ identify the rear and front axles of the train respectively. The Table also lists the individual axle positions relative to the rearmost axle $\left(\Delta x_{j}\right)$ and the axle loads $\left(2 P_{z, j}\right)$. In the analysis, longitudinal axle loads were taken as $25 \%$ of the vertical loads, i.e., $P_{X, j}=0.25 P_{Z, j}$; the spring constants were $k_{Z}=50 \mathrm{MPa}$ and $k_{X}=15 \mathrm{MPa}$. 
The track responses were evaluated at the origin of the coordinate system by considering a collection of 26 force-pairs applied at locations corresponding to the axles. With respect to the origin, at a particular instant of time, the $j^{\text {th }}$ axle was located at a distance of $x+\Delta x_{j}$. This implies that $x$ denotes the distance between the rear axle (for which $j=1, \Delta x_{j}=0$ ) and the evaluation point. The derived expressions were:

$$
\begin{aligned}
& S_{Z}^{\text {train }}=s \gamma+\frac{s \beta_{Z}}{2} \sum_{j=1}^{26} P_{Z, j} e^{-\beta_{Z}\left|x+\Delta x_{j}\right|}\left(\cos \left(\beta_{Z}\left(x+\Delta x_{j}\right)\right)+\sin \left(\beta_{Z}\left|x+\Delta x_{j}\right|\right)\right) \\
& S_{X}^{\text {train }}=\frac{s \beta_{X}}{2} \sum_{j=1}^{26} P_{X, j} e^{-\beta_{X}\left|x+\Delta x_{j}\right|} \\
& \sigma_{N}^{\text {train }}=-\frac{1}{2 A} \sum_{j=1}^{26} P_{X, j} e^{-\beta_{X}\left|x+\Delta x_{j}\right|} \operatorname{sgn}\left(\mathrm{x}+\Delta x_{j}\right)
\end{aligned}
$$

wherein $S_{Z}^{\text {train }}$ and $S_{X}^{\text {train }}$ are the vertical and longitudinal forces at the base of the $i^{\text {th }}$ sleeper, and $\sigma_{N}^{\text {train }}$ is the axial stress at the beam's neutral axis.

\section{Table 1}

Thalys HST train configuration and axle loads. ${ }^{34}$

\begin{tabular}{|c|l|l|l|}
\hline \multicolumn{1}{|c|}{ Train units } & \multicolumn{1}{|c|}{$\begin{array}{c}\text { Axle } \\
\text { indices }\end{array}$} & \multicolumn{1}{|c|}{$\begin{array}{c}\text { Axle positions with reference } \\
\text { to rearmost axle } \Delta x_{j}[\mathrm{~m}]\end{array}$} & $\begin{array}{c}\text { Axle weights } \\
{[\mathrm{kN}]}\end{array}$ \\
\hline $2 \times$ Locomotives & $\begin{array}{l}j=1 \ldots 4 ; \\
j=23 . . .26\end{array}$ & $\begin{array}{l}0.0,3.0,14.017 .0 ; \\
176.0,179.1,190.1,193.1\end{array}$ & $2 P_{Z, j}=170$ \\
\hline $2 \times$ Outer carriages & $\begin{array}{l}j=5 . . .7 ; \\
j=20 \ldots 22\end{array}$ & $\begin{array}{l}20.3,23.3,39.0 ; \\
154.2,169.9,172.9\end{array}$ & $2 P_{Z, j}=145$ \\
\hline $6 \times$ Central carriages & $j=8 \ldots 19$ & $\begin{array}{l}42.0,57.7,60.7,76.4,79.4,95.1,98.1, \\
113.8,116.8,132.5,135.5,151.2\end{array}$ & $2 P_{Z, j}=170$ \\
\hline
\end{tabular}

Figure 6 depicts calculated model responses based on equations (15) - (17) with $x$ varying from $-250 \mathrm{~m}$ to $+50 \mathrm{~m}$. Four charts are included, illustrating $S_{Z}^{\text {train }}, S_{X}^{\text {train }}, \sigma_{N}^{\text {train }}$, and the ratio $\mu_{D}^{\text {train }}=S_{X}^{\text {train }} / S_{Z}^{\text {train }}$, i.e., the friction demand at the base of a sleeper. The horizontal dashed lines 
appearing in the charts are the corresponding peak responses computed with the same parameters but for a single axle with $2 P_{Z}=170 \mathrm{kN}$ and $2 P_{X}=42.5 \mathrm{kN}$ (see Figure 4).

Figure 6(a) shows the vertical force $S_{Z}^{\text {train }}$ at the base of the $i^{\text {th }}$ sleeper as per equation (15). There are 26 distinct peaks in this chart, each corresponding to an axle; this indicates that load interaction across axles is not very pronounced. The relative difference in the peak force magnitudes for a full train and a single axle is a direct measure of the load interaction. In the case shown, the peak forces for the single axle and the full train are $32 \mathrm{kN}$ (dashed line) and $31 \mathrm{kN}$ respectively; amounting to 3\% load interaction. Based on equation (3), this peak force translates to a vertical beam displacement of about $1.0 \mathrm{~mm}$.

\section{“[insert Figure 6.]"}

Figure 6(b) shows longitudinal force $S_{X}^{\text {train }}$ at the base of the $i^{\text {th }}$ sleeper as per equation (16). Here, there are no sharp peaks corresponding to the individual axles as in Figure 6(a). This indicates a pronounced interaction across axles. The effect can be visualized in the Figure by observing that the force rises or decays over long intervals outside the length of the train (i.e., $-250<x<-200$ and $0<x<50$ ). This wide-ranging interaction is also the reason why forces are highest when the train ends are closest to the sleeper. For these train locations, the axles are more densely spaced as compared to the rest of the train. Contrasting the peak longitudinal force $(2.2 \mathrm{kN})$ with the single axle case (0.6 kN, see dashed line), provides another clear manifestation of the load interaction; this amounts to an interaction level of $266 \%$. Based on equation (10), this peak force translates to a longitudinal beam displacement of about $0.25 \mathrm{~mm}$.

Figure 6(c) presents the longitudinal stresses in the beam's neutral axis at the origin. In this case, it is noted that the beam exhibits several stress reversals during the train passage. There are 39 stress reversals whereas the number of axles crossing it are 26; indicating the prominence of the interaction effects. The magnitude of the peak stress is noted to be $3.3 \mathrm{MPa}$, which is more than two times higher than the single axle case (magnitude of about 1.4 MPa, indicated by dashed lines).

Finally, Figure 6(d) depicts the friction demand at a base of the $i^{\text {th }}$ sleeper. It shows an erratic and fluctuating demand reflecting both the vertical and longitudinal force behaviors from Figures 6(a) and 6(b). The demand consists of only 22 peaks which do not directly correspond to axle locations. The maximal friction demand occurs twice, when either of the train end units are close to the sleeper; specifically when $x=-181.75 \mathrm{~m}$ (i.e., sleeper is between axles 24 and 25) and 
$x=-11.35 \mathrm{~m}$ (i.e., sleeper is between axles 2 and 3). These maxima represent the most adverse combination of high longitudinal force and low vertical force. It should be noted that they are influenced by the choice of the spring constants, the ratio of $P_{X, j} / P_{Z, j}$, and the unit weight of the beam. Compared to the single axle case (dashed line at a value of 1.0), the peak friction demand due to the entire train is seen to be 3.5 times larger, further highlighting the interaction effects encountered for a full train. The above analysis was also repeated with a heavy sleeper, in which case $S_{Z}^{\text {train }}$ retained the same shape while the peak shifted upwards by $1.47 \mathrm{kN}$ representing the added self-weight of the system. The $S_{X}^{\text {train }}$ and $\sigma_{N}^{\text {train }}$ responses remained unaltered, while the maximal friction demand dropped to 0.9 .

\section{Conclusions}

This paper focused on modeling railway track responses to braking loads. An analytical framework was proposed based on an infinite Euler-Bernoulli beam supported by an orthogonal Winkler foundation, subjected to concentrated or distributed forces (or as a combination), in both vertical and longitudinal directions. Such modeling applies to different track types, and can address both traditional and modern braking systems. Closed-form expressions were provided for calculating diverse set of responses such as: (i) longitudinal rail stresses and displacements, (ii) vertical and longitudinal displacements or forces at a sleeper base (or fastener), and (iii) friction demand at a sleeper base (or fastener) corresponding to zero slippage condition.

Subsequently, for a range of realistic parameters, the model was first demonstrated by application to a single axle. The specific findings from this demonstration were: (i) peak longitudinal rail stress was independent of the support condition but its decay was a function of the latter, (ii) longitudinal track responses were spread over a much longer zone in comparison to vertical track responses, (iii) peak friction demand exhibited a positive relationship with the spring constants, and (iv) peak friction demand was unrealistically high for a standard sleeper weight and acceptably low for a heavier sleeper. The model was demonstrated next for a full train considering a traditional braking system. This analysis showed that responses in the longitudinal direction, as well as peak friction demand, increased considerably compared to the single axle case. This outcome was due to the pronounced load interaction in the longitudinal direction. 
These findings can be generalized to arrive at the following conclusions for a braking event: (i) for a full train, all track responses involving longitudinal loads cannot be deduced from a single axle analysis; calculations must be done on a case-by-case basis as all related peak responses are expected to be larger, (ii) for track types in which sliding resistance of the sleepers is solely supplied by a frictional mechanism at the base, a heavy sleeper type may be needed, (iii) given that forces in the sleepers and fasteners are interlinked, when sleepers have higher sliding resistance, greater demand will be placed on fasteners to resist rail pullout.

In future studies, the proposed framework may be employed to address additional cases involving, e.g.: (i) modern braking technologies, (ii) uneven braking conditions between two rails or along a single rail, and (iii) effects of vertical track gradient, i.e., uphill or downhill. Moreover, the framework capabilities may be enhanced further by: (i) considering second-order effects from coupling of responses in the two directions; (ii) utilizing a Pasternak foundation for the vertical track responses, and (iii) including dynamic (inertia) effects in the formulation. Nonetheless, even in its current form, the proposed approach is deemed flexible, robust, and well suited for analyzing track responses to decelerating vehicular loads.

\section{Acknowledgement}

The support from Innovation Fund Denmark is gratefully acknowledged. This study is part of 'Roads2Rails: Innovative and cost-effective asphalt based railway construction system' (Grand Solutions 5156-00006B). The authors would like to thank all the project participants for their valuable contributions.

\section{References}

1. UIC. A global vision for railway development. International Union of Railways, ISBN 978-27461-2449-3, 2015.

2. Koseki T. Technical trends of railway traction in the world. Proceedings of the 2010 International Power Electronics Conference (IPEC), IEEE, 2010; 2836-2841.

3. Cruceanu C. Train braking, Reliability and safety in railway. Perpinya, X. (Ed.). InTech 2012; 29-74.

4. Wang P.J and Chiueh S.J. Analysis of eddy-current brakes for high speed railway. IEEE Transactions on Magnetics 1998; 34(4): 1237-1239.

5. Domingo L.M., Herraiz J.I.R., Zamorano C., and Herraiz T.R. Design of a new high lateral resistance sleeper and performance comparison with conventional sleepers in a curved railway track by means of finite element models. Latin American Journal of Solids and Structures 2014; 11(7): 1238-1250. 
6. Manalo A., Aravinthan T., Karunasena W., and Ticoalu A. A review of alternative materials for replacing existing timber sleepers. Composite Structures 2010; 92(3): 603-611.

7. Nimbalkar S., Indraratna B., Dash S.K., and Christie D. Improved performance of railway ballast under impact loads using shock mats. Journal of Geotechnical and Geoenvironmental Engineering 2012; 138(3): 281-294.

8. Schneider P., Bolmsvik R., and Nielsen J.C. In situ performance of a ballasted railway track with under sleeper pads. Proceedings of the Institution of Mechanical Engineers, Part F: Journal of Rail and Rapid Transit 2011; 225(3): 299-309.

9. Egana J.I., Vinolas J., and Seco M. Investigation of the influence of rail pad stiffness on rail corrugation on a transit system. Wear 2006; 261(2): 216-224.

10. Woodward P.K., Kennedy J., Medero G.M. and Banimahd M. Application of in situ polyurethane geocomposite beams to improve the passive shoulder resistance of railway track. Proceedings of the Institution of Mechanical Engineers, Part F: Journal of Rail and Rapid Transit 2012; 226(3): 294-304.

11. Esmaeili M., Amiri S., and Jadidi K. An investigation into the use of asphalt layers to control stress and strain levels in railway track foundations. Proceedings of the Institution of Mechanical Engineers, Part F: Journal of Rail and Rapid Transit 2014; 228(2): 182-193.

12. Rose J. and Anderson J. Long-term performance of asphalt underlayment trackbeds for special trackbed applications. In Proceedings, American Railway Engineering and Maintenance-of-Way Association (AREMA) Annual Conference and Exposition at Kentucky 2006.

13. Teixeira P.F, López-Pita A., Casas C., Bachiller A., and Robuste F. Improvements in high-speed ballasted track design: Benefits of bituminous subballast layers. Transportation Research Record: Journal of the Transportation Research Board 2006; 1943: 43-49.

14. Gautier P.E. Slab track: Review of existing systems and optimization potentials including very high speed. Construction and Building Materials 2015; 92: 9-15.

15. Huang Y.H., Rose J.G., and Khoury C.J. Thickness design for hot-mix asphalt railroad trackbeds. Annual Journal AAPT, Miscellaneous 1987; 56(87): 427-453.

16. Rose J.G., Brown E.R., and Osborne M.L. Asphalt trackbed technology development: the first 20 years. Transportation Research Record: Journal of the Transportation Research Board 2000; 1713: 1-9.

17. Lee S.H., Choi Y.T., Lee H.M., and Park D.W. Performance evaluation of directly fastened asphalt track using a full-scale test. Construction and Building Materials 2016; 113: 404-414.

18. Esmaeili M., Heydari-Noghabi H., and Sayadi A. Field investigation on the lateral resistance of railway tracks including hot mix asphalt layer. Road Materials and Pavement Design 2016; DOI: 10.1080/14680629.2016.1250666.

19. De Iorio A., Grasso M., Penta F., Pucillo G.P., Rossi S., \& Testa M. On the ballast-sleeper interaction in the longitudinal and lateral directions. Proceedings of the Institution of Mechanical Engineers, Part F: Journal of Rail and Rapid Transit 2016; DOI: 10.1177/0954409716682629.

20. Wang W.J., Shen P., Song J.H., Guo J., Liu Q.Y., and Jin X.S. Short communication: experimental study on adhesion behavior of wheel/rail under dry and water conditions. Wear 2011; 271: 2699-2705. 
21. Tran M.T., Ang K.K., Luong V. H., and Dai J. High-speed trains subject to abrupt braking. Vehicle system dynamics 2016; 54(12): 1715-1735.

22. Van M.A. Stability of continuous welded rail track. Ph.D. Dissertation, TU Delft, The Netherlands 1997.

23. Rhodes D., Maree J.S., and Barthram P. Track design for extreme traction and braking forces. In 8th International Heavy Haul Association Conference (IHHA), Rio de Janeiro, RJ, Brazil. 2005.

24. Zhang Z., Andrawes B., and Edwards J.R. Parametric study on the distribution of longitudinal load in railway track under dynamic wheel loading using finite element analysis. SSRG International Journal of Civil Engineering 2015; 2(5): 28-41.

25. Hetényi M. Beams on elastic foundation: theory with applications in the fields of civil and mechanical engineering. Eleventh printing, Ann Arbor, The University of Michigan Press, 1979.

26. Hosking R.J. and Milinazzo F. Floating ladder response to a steadily moving load. Mathematical Methods in the Applied Sciences 2007; 30: 1823-1841.

27. Esveld C. Modern railway track. Zaltbommel, The Netherlands, MRT-productions, 2001.

28. UIC Code 774-3R.Track-bridge interaction recommendations for calculations. Second Edition, International Union of Railways, Paris, France, 2001.

29. Wang W.J., Shen P., Song J.H., Guo J., Liu Q.Y., and Jin X.S. Short communication: experimental study on adhesion behavior of wheel/rail under dry and water conditions. Wear 2011; 271: 2699-2705.

30. Stewart H.E. Measurement and prediction of vertical track modulus. Transportation Research Record 1985; 1022: 65-71.

31. Selig E.T. and Li D. Track modulus: its meaning and factors influencing it. Transportation Research Record 1994; 1470: 47-54.

32. Le Pen L.M. and Powrie W. Contribution of base, crib, and shoulder ballast to the lateral sliding resistance of railway track: a geotechnical perspective. Proceedings of the Institution of Mechanical Engineers, Part F: Journal of Rail and Rapid Transit 2011; 225(2): 113-128.

33. Kish A. On the fundamentals of track lateral resistance. The AREMA 2011 Annual Conference, Minneapolis, USA, September 18-21, 2011.

34. Degrande G. and Schillemans L. Free field vibrations during the passage of a Thalys high-speed train at variable speed. Journal of Sound and Vibration 2001; 247(1): 131-144. 


\section{Figures}

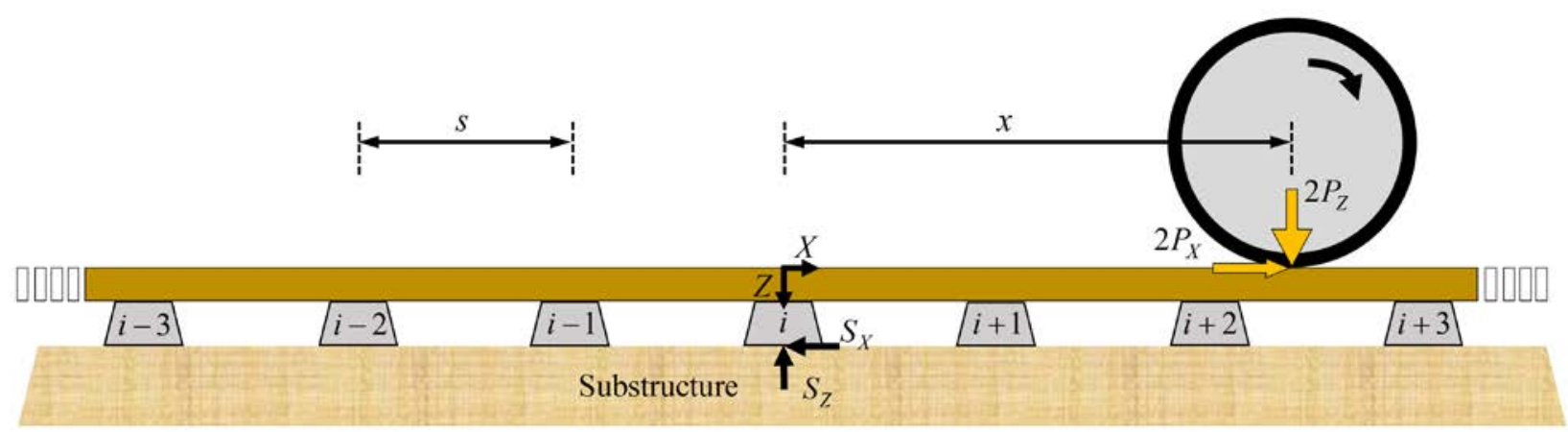

Figure 1. A sketch depicting a single train axle decelerating over a rail supported on sleepers.

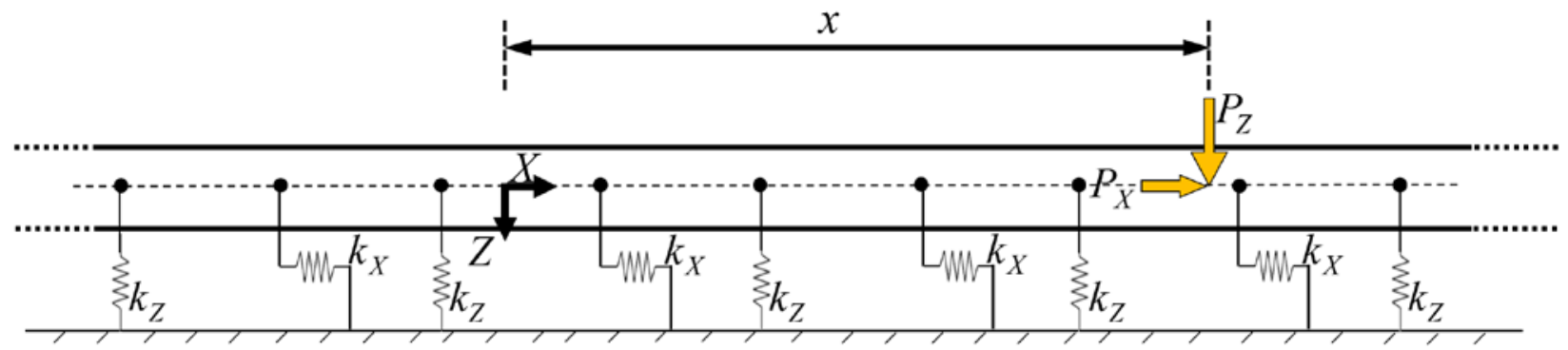

Figure 2. An orthogonal Winkler foundation model for simulating braking of a single wheel.

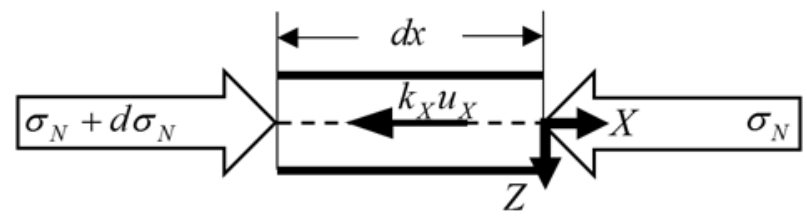

Figure 3. Magnified view of induced axial stresses in an infinitesimal beam element. 


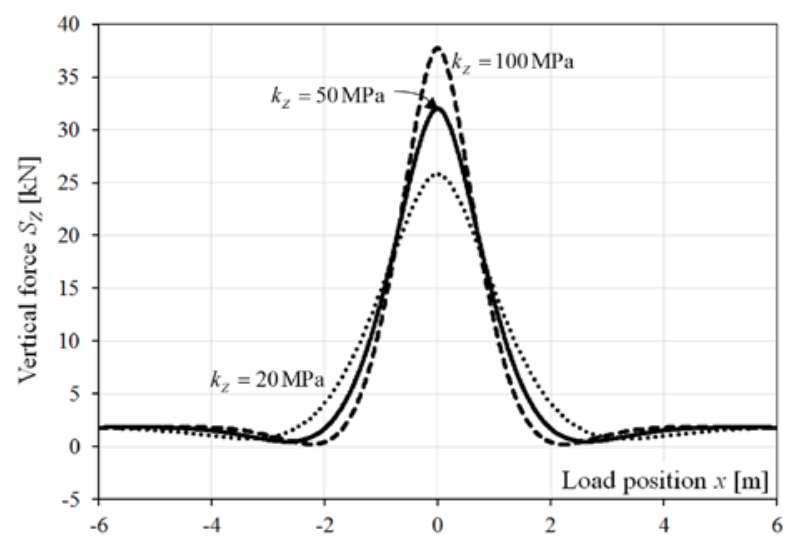

(a)

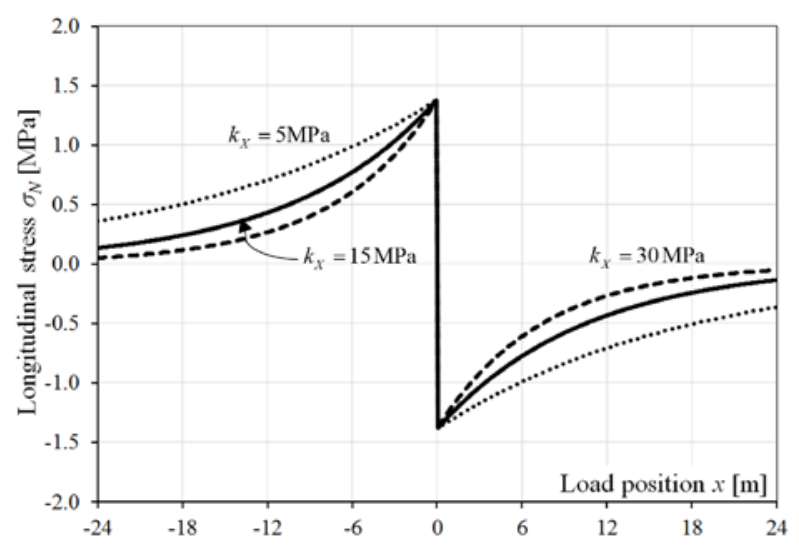

(c)

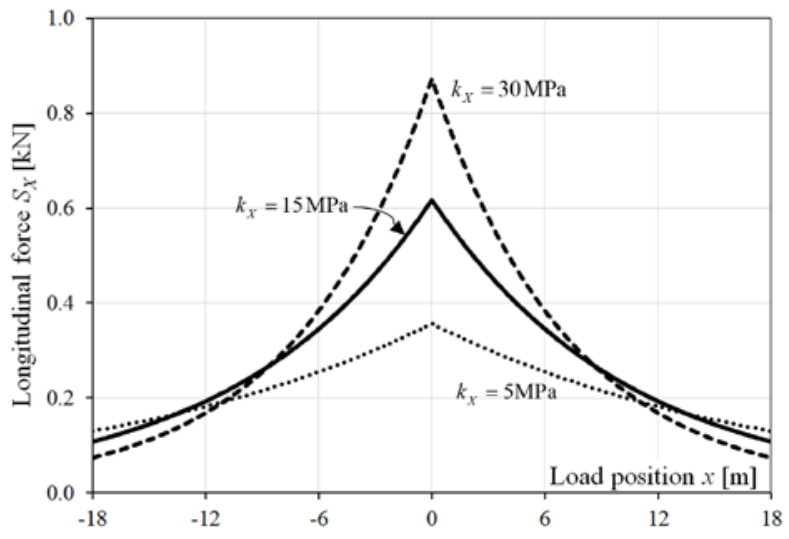

(b)

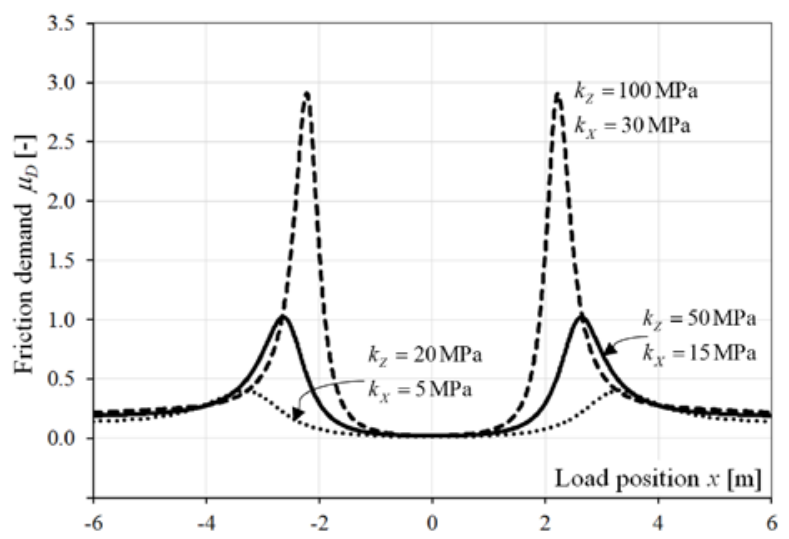

(d)

Figure 4. Model responses for a single axle as a function of the load positions: (a) vertical force at the base of a sleeper and (b) longitudinal force at the base of a sleeper, and (c) longitudinal stress at the beam's neutral axis and (d) friction demand at the base of a sleeper.

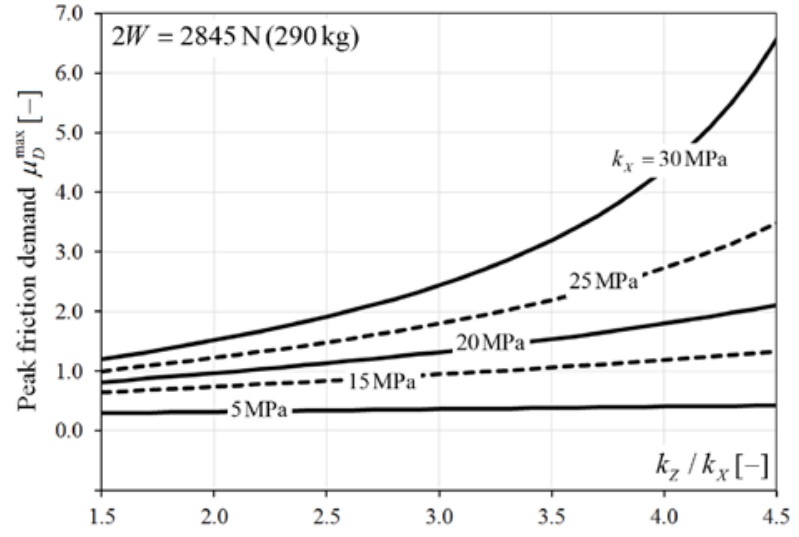

(a)

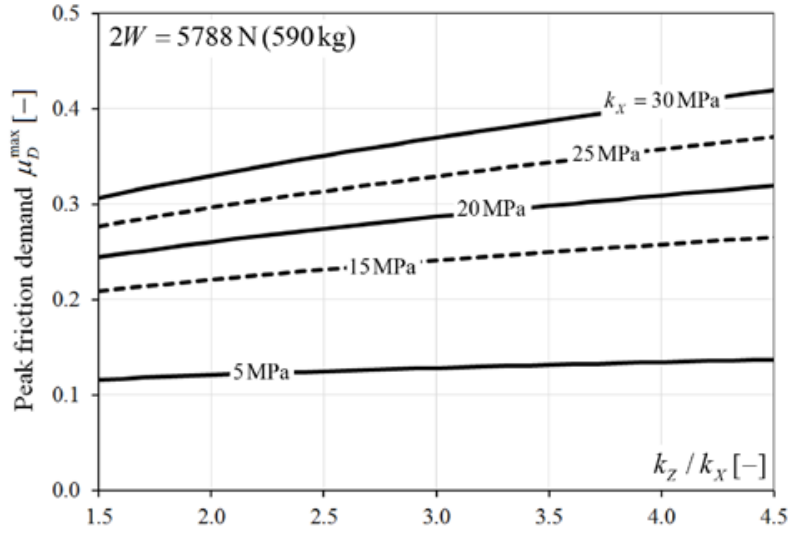

(b)

Figure 5. Peak friction demand as a function of spring constant ratios for different values of longitudinal spring constants: (a) standard sleeper and (b) heavy sleeper. 


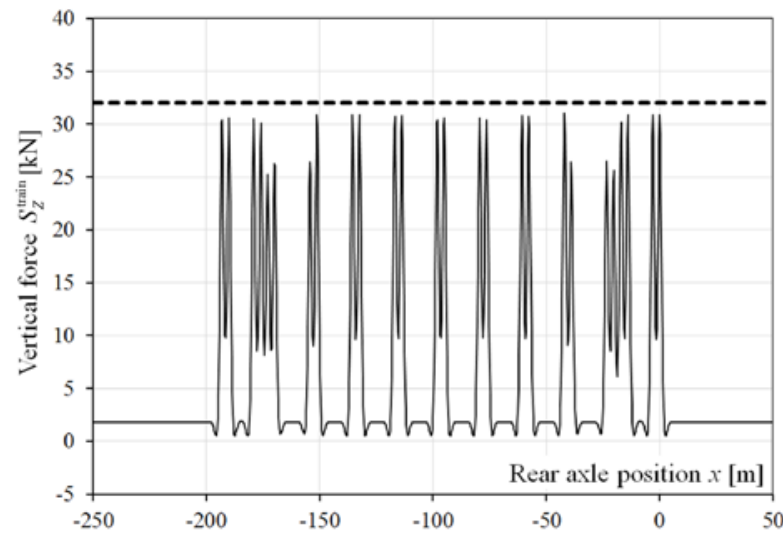

(a)

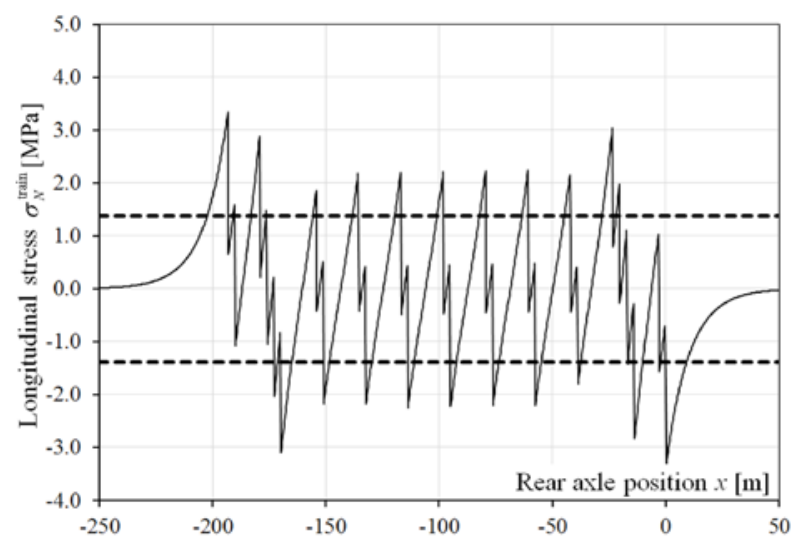

(c)

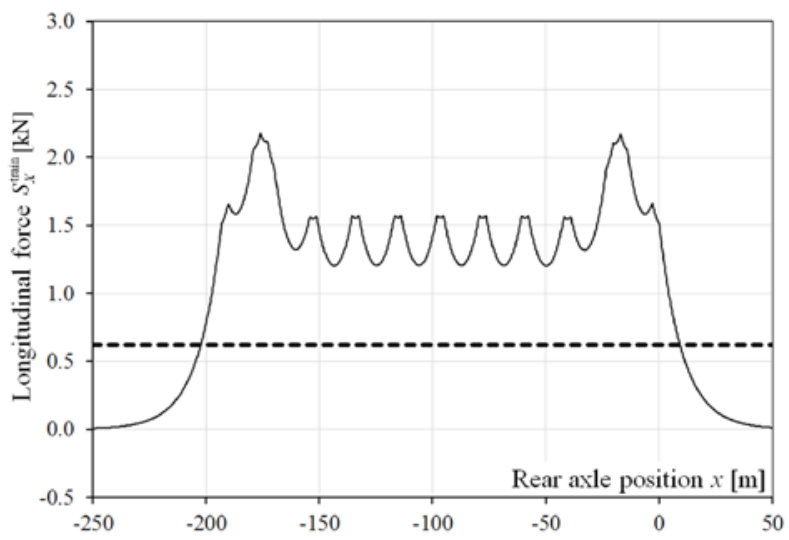

(b)

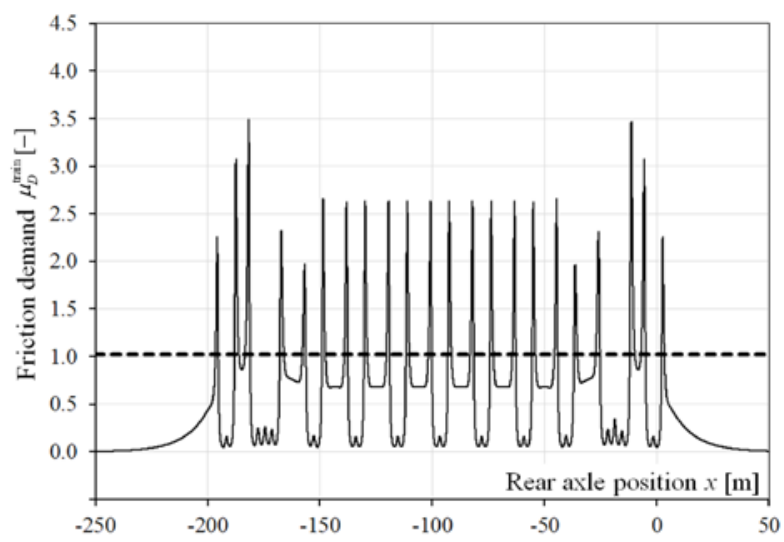

(d)

Figure 6. Model responses for a full train as a function of the distance from rear axle: (a) vertical force at the base of a sleeper and (b) longitudinal force at the base of a sleeper, and (c) longitudinal stress at beam's neutral axis and (d) friction demand at the base of a sleeper. 